\title{
Análise da propagação e manutenção dos vórtices gerados por um mi- croburst estático e isolado
}

\author{
Analysis of the propagation and maintenance of vortices generated by a static and \\ isolated microburst
}

\section{Giuliano Demarco ${ }^{1}$, Vagner Anabor ${ }^{2}$, Umberto Rizza ${ }^{3}$, Franciano Scremin Puhales², Luís Gustavo Nogueira Martins², Felipe Denardin Costa ${ }^{4}$, Otávio Costa Acevedo ${ }^{2}$}

\author{
${ }^{1}$ Pós Doutorando em Física e Professor Voluntário no Departamento de Física da Universidade Federal de Santa \\ Maria, RS, Brasil \\ giulianofisico@gmail.com \\ ${ }^{2}$ Professor do Departamento de Física do Centro de Ciências Naturais e Exatas da Universidade Federal de Santa \\ Maria, RS, Brasil \\ vanabor@gmail.com; franciano.puhales@ufsm.br; lgnm.sm@gmail.com; otavio@ufsm.br \\ ${ }^{3}$ Consiglio Nazionale delle Ricerche, Istituto Di Scienze Dell Atmosfera e Del Clima, Itália \\ urizza@gmail.com \\ ${ }^{4}$ Professor da Universidade Federal do Pampa , RS, Brasil \\ fdenardin@gmail.com
}

\begin{abstract}
Resumo
A Região Sul do Brasil é especialmente atingida por eventos meteorológicos extremos. Frequentemente rajadas de vento intensas oriundas da convecção profunda podem se desenvolver como Microbursts, produzindo ventos superiores a $100 \mathrm{~km} / \mathrm{h}$. Na tentativa de compreender os processos físicos e dinâmicos envolvidos neste fenômeno, um microburst estático e isolado foi produzido através da simulação de grandes turbilhões. Uma análise qualitativa da propagação e manutenção do anel de vorticidade gerado pelo microburst é realizada com o objetivo de entender sua evolução.
\end{abstract}

Palavras-chave: Vorticidade, Simulação dos grandes turbilhões, microburst.

\begin{abstract}
The Southern Brazilian region is specially affected by extreme weather events, very often intense wind gusts coming from deep convection may develop it self as a microburst producing winds higher than $100 \mathrm{~km} / \mathrm{h}$. In order to understand the physical and dynamical process evolved in this phenomena, a static and isolated microburst is produced trough a Large Eddy Simulation. A quantitative analysis of propagation an maintenance of the microburst vortex ring is performed in order to understand its evolution.
\end{abstract}

Keywords: Vorticity, large-eddy simulation, microburst. 


\section{Introdução}

Eventos meteorológicos severos têm causado grande preocupação para a população em geral e têm sido cada vez mais comuns no Brasil. Também provocam grandes destruições no meio socioeconômico e ambiental. Consequentemente, torna-se necessário um estudo mais aprofundado a respeito destes fenômenos, desde sua formação até os impactos resultantes de tais acontecimentos.

Devido à previsão do tempo no seu estado atual de desenvolvimento, obter resultados apenas satisfatórios por um período muito curto de tempo, não é possível de detectar remotamente e com precisão mudanças bruscas de circulação de ar que são tão importantes, por exemplo, para segurança da aviação.

Um fenômeno de grande interesse para áreas como meteorologia, engenharia e aviação civil é o Downburst (Fujita, 1985, 1990; Wakimoto, 2001). Este fenômeno pode ser descrito por um fluxo descendente intenso originado por uma tempestade (ou sistema de tempestades). Após esta colidir com o solo em uma área localizada, intensos movimentos divergentes de ventos são gerados. Esses ventos podem chegar a mais de 30 $\mathrm{ms}^{-1}$ e se propagar lateralmente por uma área de até várias dezenas de quilômetros de raio. Eles apresentam forte cisalhamento em pequenas escalas espaciais, o que os torna perigosos e catastróficos para o desempenho de aviões em baixas altitudes, especialmente, durante pousos e decolagens (Fujita, 1985, 1990). Tal fenômeno pode ser redefinido como microburst ou macroburst. Para tanto, deve-se levar em consideração as extensões laterais e o tempo de duração. No caso de um microburst, tais escalas são menores que $4 \mathrm{~km}$ e o tempo de duração do evento está em torno de 2 e 5 minutos (Proctor, 1988; Wakimoto, 2001). Quando o raio do escoamento divergente for superior a $4 \mathrm{~km}$, e possuir um tempo de duração maior que 5 minutos, o fenômeno é por vezes referido como macroburst

Uma característica interessante de um microburst é a formação de um vórtice que se expande radialmente logo depois que a corrente de ar descendente toca o solo (Fujita, 1990). Compreender como esse vórtice evolui é um aspecto importante para analisar a evolução da dinâmica de um microburst.

Distintos trabalhos relacionados ao estudo de $m i$ croburst, tem sido desenvolvido no decorrer dos anos como: trabalhos de campo (Fujita, 1985; Hjelmfelt, 1988; Weisman, 1993). Porém, fica difícil extrair conclusões a respeito de suas propriedades globais devido ao fato de serem muito localizados e aleatórios.

Por esse motivo, os microbursts têm sido estudados através de simulações numéricas (Proctor, 1988; Kim e Hangan, 2007; Vermeire et al., 2011; Anabor et al.,
2011) e de simulações mediante experimentos realizados em laboratórios (Landreth e Adrian, 1990; Lundgren et al., 1992; Mason et al., 2005; Demarco et al., 2013) e ambas têm sido amplamente utilizados para o estudo da dinâmica de microbursts.

Independente do tipo de simulação de microburst, todas relatam a formação de um vórtice primário orientado horizontalmente na borda de saída do escoamento (Proctor, 1988; Anabor et al., 2011; Demarco et al., 2013), que também é mencionado em estudos observacionais de microbursts (Wakimoto, 2001)).

Outro fato interessante, está relacionado à observação da estrutura, manutenção e propagação desse vórtice. Mantendo as perturbações no escoamento, sugere-se a possibilidade do aparecimento de um vórtice secundário (Hjelmfelt, 1987, 1988). O mesmo foi observado em simulações numéricas (Proctor, 1989; Kim e Hangan, 2007; Anabor et al., 2011) e experimentais (Mason et al., 2005; Demarco et al., 2013).

Neste trabalho, analisa-se parte de uma série de resultados de simulações de microburst realizados com LES (do inglês Large Eddy Simulation). O objetivo é aprofundar a análise da condução do microburst, como escoamento, formação e propagação de vórtices primários e secundários. Ademais, como a manutenção do vórtice secundário e as magnitudes dos ventos de saída referentes ao fenômeno.

\section{Simulação de microburst}

Para a realização da simulação utilizou-se o código proposto por Moeng (1984) e a parametrização de sub-grade de Sullivan et al. (1994). Foi utilizado um domínio espacial de $(x 1 ; x 2 ; x 3)=(10 \mathrm{~km} ; 10 \mathrm{~km} ; 2 \mathrm{~km})$ sendo as duas primeiras horizontais e a terceira vertical. O domínio computacional consiste em uma grade de $128 \times 128 \times 128$ pontos em cada dimensão espacial. Portanto, o espaçamento da grade é dado por $(x=78,125, y=78,125, z=15,625)$.

Simulações de microburst, numéricas ou experimentais, são baseadas em duas abordagens principais, no que diz respeito ao mecanismo de condução que inicia uma corrente de gravidade usada para representar o microburst. A primeira é aproximação por jato incidente (Landreth e Adrian, 1990; Mason et al., 2005; Kim e Hangan, 2007) e a segunda, por fonte de resfriamento (Proctor, 1988; Lundgren et al., 1992; Vermeire et al., 2011; Anabor et al., 2011).

No presente estudo, a flutuabilidade negativa que impulsiona a formação microburst é parametrizado através da imposição de um termo fonte adicional na equação para a temperatura potencial virtual.(Anabor et al., 2011). 


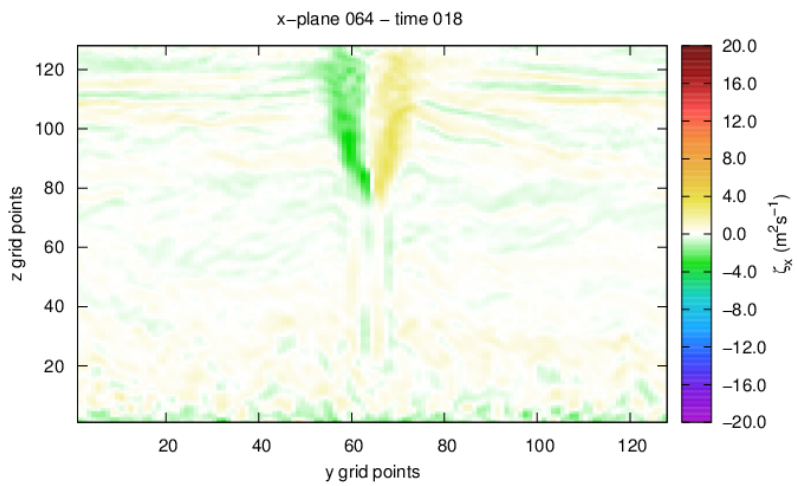

(a) Instante 18 segundos

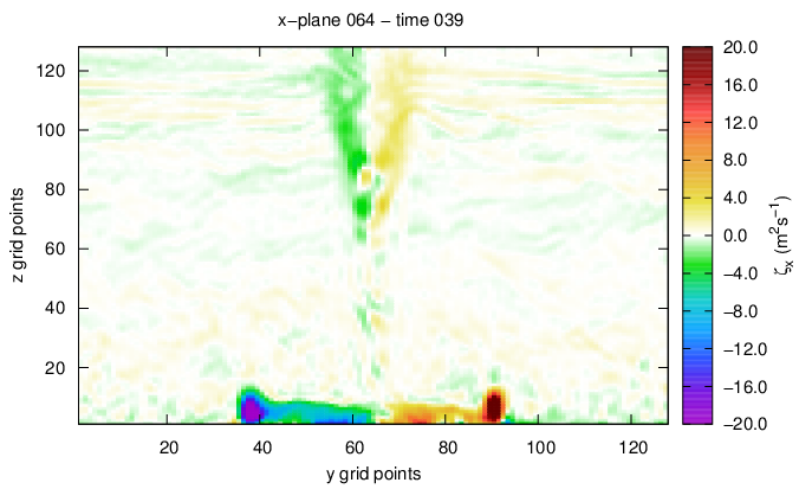

(c) Instante 39 segundos

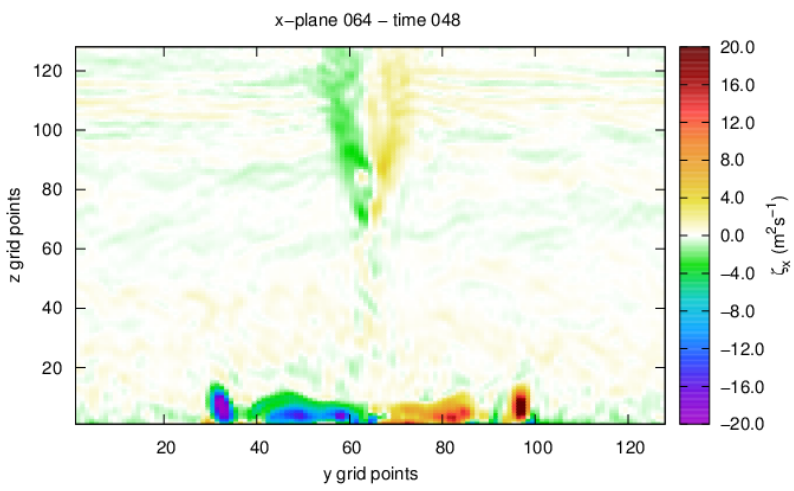

(e) Instante 48 segundos

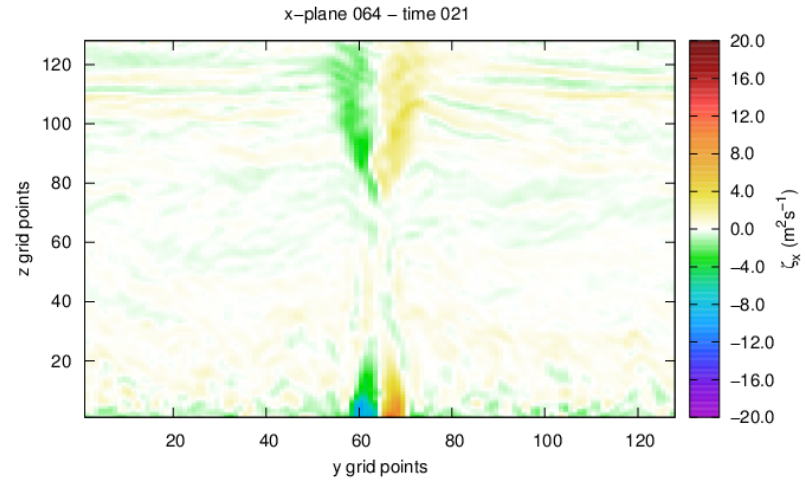

(b) Instante 21 segundos

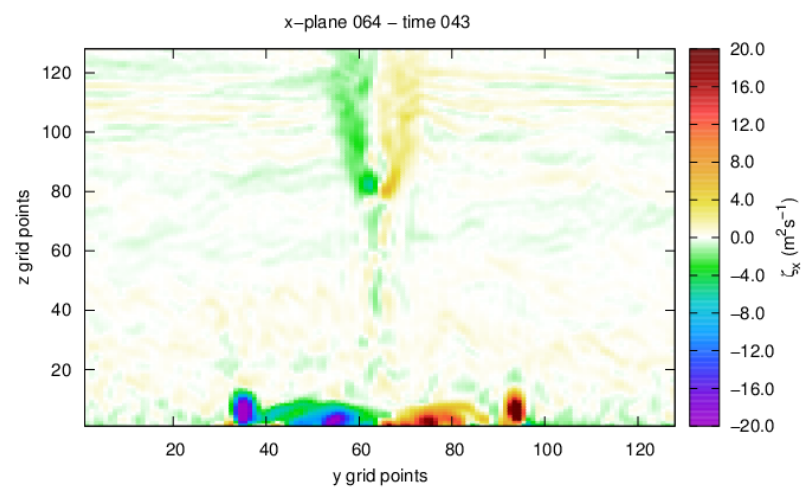

(d) Instante 43 segundos

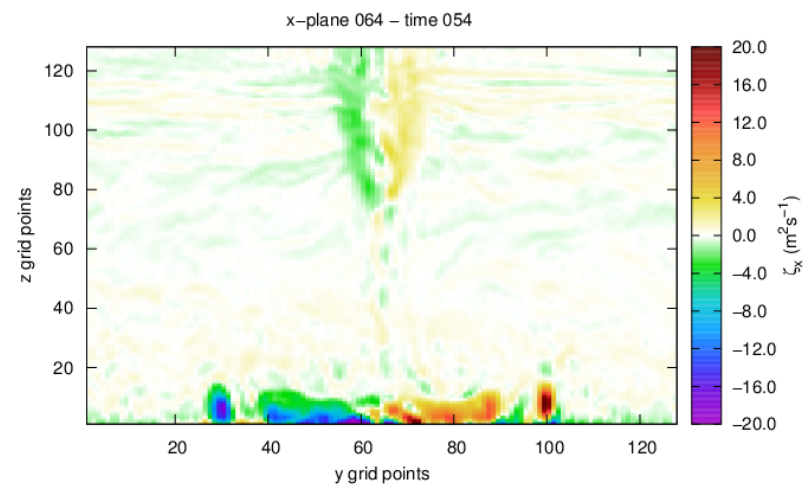

(f) Instante 54 segundos

iigura 1: Seis quadros temporais distintos para evolução do campo de vorticidade. Em todos os instantes, as análises oram feitas no centro do domínio. 


\section{Resultados e discuções}

Simultaneamente ao aparecimento do fluxo descendente frio, ocorre o desenvolvimento de vórtices na vizinhança do eixo principal e que acompanham a extensão vertical do modelo. Estes vórtices tem origem no cisalhamento entre o fluxo vertical descendente e o ambiente médio. Quando o jato frio toca o solo ocorre a formação de vórtices que se propagam horizontalmente pelo domínio. Os resultados das simulações mostram que à medida que o fluxo toca o solo, a formação de um ponto de singularidade realiza a transferência de momento vertical para um fluxo divergente e horizontalmente acelerado. $\mathrm{Na}$ extremidade deste fluxo um anel de vorticidade começa a se desenvolver como resposta ao forte gradiente horizontal de flutuabilidade existente através da borda do outflow Orf et al. (1996). A figura 1 apresenta seis quadros temporais distintos para evolução do campo de vorticidade. Em todos os instantes, as análises foram feitas no centro do domínio. Nos primeiros quatro quadros temporais apresentados, fica clara a formação de um vórtice principal (definido na literatura como primário, 1(a), 1(b), 1(c) e 1(d) ). Nos instantes seguintes, é possível visualizar a formação de novos vórtices (1(e) e 1(f)). No decorrer do tempo, o vórtice primário cresce significativamente atingido uma altura de aproximadamente $280 \mathrm{~m}$ e, desloca-se radialmente durante todo o período. Além disso, são observadas vorticidades negativas geradas logo à frente do vórtice (formação de dipolos). Resultados similares foram obtidos para outras condições experimentais testadas (Demarco et al., 2013). Outra análise importante esta relacionada à manutenção e organização dos vórtices. Mantendo-se o fluxo vertical, ocorre a formação de novos vórtices, porém, com uma estrutura vertical de menor magnitude. Ademais, os vórtices subsequentes possuem uma estrutura menos organizada. Isso se deve, provavelmente, à diferença de densidade entre a coluna descendente e a atmosfera que acaba diminuindo devido à mistura (1(e) e 1(f)). Quanto maior a diferença de densidade entre o fluido da coluna descendente e a atmosfera, maior é a organização e a manutenção dos vórtices (Demarco et al., 2013).

\section{Conclusões}

A simulação de um microburst estático e isolado foi efetuada utilizando a técnica de simulação dos grande turbilhões. Inicialmente, os resultados obtidos são compatíveis com as observações do campo de vento realizadas durante Microbursts pois, apresentam um anel de vorticidade que se propaga radialmente para fora do epicentro do evento, sendo sucedido por um segundo e um terceiro anel de vorticidade que ocorre após a reintensificação do gradiente horizontal de pressão.
Ademais, os resultados obtidos mostram incialmente, de forma qualitativa, a dependência da diferença de densidade entre a coluna descendente e a atmosfera. Conforme o tempo evolui, o segundo e o terceiro vórtice que se forma, após a passagem do primário, possuem um alcance vertical menor e se desprendem rapidamente, se compararmos com o primário, que possui um maior deslocamento horizontal. Isso pode ser explicado por haver uma menor diferença de densidades entre os fluidos.

\section{Agradecimentos}

Os autores deste trabalho agradecem ao CNPq (Conselho Nacional de Desenvolvimento Cientifico e Tecnologico, Brasil), CAPES (Coordenação de Aperfeiçoamento de Pessoal de Nível Superior) e CNR (Consiglio Nazionale delle Ricerche, Italia) por financiarem este trabalho.

\section{Referências}

Anabor, V., Rizza, U., Nascimento, E. L., Degrazia, G. A. (2011). Large-eddy simulation of a microburst. Atmospheric Chemistry and Physics, 11, 9323 - 9331.

Demarco, G., Barrere, N., Sarasúa, G., Martí, A., Acevedo, O., Nascimento, E., Cabeza, C. (2013). Combined effect of jet impingement and density perturbation forcing on the evolution of laboratory-simulated microbursts. Journal of Wind Engineering and Industrial Aerodynamics, 123, 69-76.

Fujita, T. (1985). The downburst, microburst and macroburst, sattellite and mesometeorology research project (smrp). Dept of Geophisycal Science, 210, 122p.

Fujita, T. (1990). Downbursts: meteorological features and wind field characteristics. Journal of Wind Engineering and Industrial Aerodynamics, 36, 75-86.

Hjelmfelt, M. (1987). The microburst of 22 june 1982 in jaws. Journal of the Atmospheric Sciences, 44(12), 16461665.

Hjelmfelt, M. (1988). Structure and life cycle of microburst outflows observed in colorado. Journal of Appllied Meteorology, 27, $900-927$.

Kim, J., Hangan, H. (2007). Numerical simulations of impinging jets with application to downbursts. Journal of Wind Engineering and Industrial Aerodynamics, 95, 279 -298 .

Landreth, C. C., Adrian, R. J. (1990). Impingement of a low reynolds number turbulent circular jet onto a flat plate at normal incidence. Experiments in Fluids, 9, 74 84. . 
Lundgren, T., Yao, J., Mansour, N. (1992). Microburst modelling and scaling. Journal of Fluid Mechanics, 239, 461 - 488 .

Mason, M., Letchforda, C., James, D. (2005). Pulsed wall jet simulation of a stationary thunderstorm downburst, part a: Physical structure and flow field characterization. Journal of Wind Engineering and Industrial Aerodynamics, 93, 557-580.

Moeng, C. H. (1984). A large-eddy-simulation model for the study of planetary boundary layer turbulence. Journal of Atmospheric Science, 41(13), 2052-2062.

Orf, L. G., Anderson, J. R., Straka, J. M. (1996). A threedimensional numerical analysis of colliding microburst outflow dynamics. Journal of Atmospheric Sciences, 53, 2490-2511.

Proctor, F. H. (1988). Numerical simulations of an isolated microburst. part i: Dynamics and structure. Journal of the atmospheric sciences, 45, 3137-3160.

Proctor, F. H. (1989). Numerical simulationsof an isolated microburst. part ii: Sensitivity experiments. Journal of the atmospheric sciences, 46, 2143-2165.

Sullivan, P. P., McWilliams, J. C., Moeng, C. H. (1994). A subgrid-scale model for large-eddy simulations of planetary boundary-layer flows. Boundary Layer Meteorology, 71(3), 247-276.

Vermeire, B. C., Orf., L. G., Savory, E. (2011). Improved modelling of downburst out flows for wind engineering applications using a cooling source approach. Journal of Wind Engineering and Industrial Aerodynamics, 99, 801-814.

Wakimoto, R. M. (2001). Convectively-driven high wind events. American Meteorological Society, 28, 255 - 298.

Weisman, M. L. (1993). The genesis of severe, long-lived bow echoes. Journal of the atmospheric sciences, 50, 645670. 